\title{
About the Method of Analysis of Economic Correlations by Differentiation of Spline Models
}

\author{
Ruslan Hizrailevich Ilyasov ${ }^{1}$ \\ ${ }^{1}$ The Chechen State University, Russia \\ Correspondence: Ruslan Hizrailevich Ilyasov, The Chechen State University, Sheripova Street, 32, Grozny, \\ Chechen Republic 364907, Russia.
}

Received: July 3, $2014 \quad$ Accepted: July 5, $2014 \quad$ Online Published: September 4, 2014

doi:10.5539/mas.v8n5p197 URL: http://dx.doi.org/10.5539/mas.v8n5p197

\begin{abstract}
The article considers spline approximation as one of efficient methods of modeling economic dynamics. Spline approximation of economic dynamics allows carrying out qualitative and accurate transition from discrete values of a lattice function to a continuous model of a process, which allows calculating values of a studied index at any time point (interpolation). Spline representation improves the quality of economic dynamics modeling while saving the real values of the studied process at each time point. In this article, differentiation of spline models is used for analysis of the economic indexes growth rate. Correlations are detected and itemized by comparison of derivatives. The possibility of detecting "latent trends" is demonstrated by differentiation of spline models of the dynamics using the example of economic indexes of the oil and gas market of Russia. For example, in the first case, we consider spline models of the dynamics of export prices for oil and natural gas. Here, the correlation of the studied indexes is obvious and is detected by both calculation of the correlation ratio and visualization of the studied rows of dynamics with spline models. As an opposite example, we consider the dynamics of the volumes of oil and natural gas export. In this case, we gain the correlation ratio close to zero, which is to evidence absence of correlation. Modeling of the studied dynamics with cubic splines also does not detect any correlation between the dynamics of volumes of the oil and gas export. Our assumptions about "latent trends" are also confirmed by differentiation of spline models - the correlation between the change rate of the volumes of the oil and gas export is detected. Use of spline functions at economic dynamics modeling is determined with such positive properties of theirs as continuity, flexibility, differentiability, the property of minimal curve, etc.
\end{abstract}

Keywords: correlation, approximation, dynamics, splines, derivative, rate, export, oil, natural gas

\section{Introduction}

Modern processes in the economy flow with permanently changing accelerations being exposed to the impact of an unstable set of factors. For analysis of correlations, the classical econometrics (Pinto, 2011) uses the popular correlation method. Depending on the value and the sign of the correlation ratio, the conclusions on presence and proximity of the correlation are made. However, the correlation ratio allows evaluating the interdependence of the studied indexes only in general terms, i.e. on the average for a whole row or a part of the row.

The values of the indexes of the economic process are usually represented in the form of discrete values of a temporal row, i.e. are described with a certain lattice function. However, the lattice, discrete, or table representations of the economic variables do not allow carrying out qualitative analysis of the continuous economic dynamics for a number of reasons, namely:

- there is no term "derivative", representing the trends of an economic process;

- the conceptual absence of interpolative "smoothing" of the lattice, i.e. the impossibility to find the values of the function within the intervals between the nodes;

- the impossibility to forecast the behavior of the economic indexes through extrapolation of the continuous analytical model;

- The impossibility to determine the phase relations in each economic process, build phase patterns, find phase parametric interdependencies between the behavior of certain indexes, etc.

For the analysis of the continuous inertial dynamics of the economic process, the necessity rises to build such a 
model, which could detect unambiguously interpreted analytical and quantitative correlations, allow deducing formal consequences from them, detect the properties, construct laws, and forecast the development of the process. Such opportunities for a researcher are found at modeling economic dynamics with splines (Wang and Yu, 2014; Oh, Kim and Hong, 2008; Sekigawa, 2011).

Splines are piecewise-polynomial functions determined on an interval $[a, b]$ and having a finite number of continuous derivatives within this interval. The word "spline" means a flexible ruler used for drawing smooth curves through the given plots on a plane.

Spline appeared in the approximation theory as auxiliary means for proving the Weierstrass theorem and direct theorems of the theory of polynomial approximations as far back as in the times of Lebesgue and Jackson. Spline-based approximations (Schoenberg, 1968) also naturally occur at researching quadrature formulas. Spline approximation (Schoenberg, 1970) was also systematically studied and actively propagated by Schoenberg (Schoenberg, 1979) in the problems of numerical mathematics (Schoenberg, 1983).

\section{Materials and Methods}

Use of spline models can efficiently complement the correlative approach to the correlations analysis. Comparison of the first two derivative cubic splines approximating the studied dynamics can detect the "latent trends" of the economic dynamics.

Mathematical splines are a set of correlated "pieces" of power polynomials, which represent a process in intervals between nodes. Splines consist of intervals of a small order polynomial, which converge in the given nodes of the process (the nodes of its lattice function). A mathematical spline of $q^{\text {th }}$ order is continuous and has a $(q-1)$ continuous derivative, $q^{\text {th }}$ derivative can be subject to discontinuity in the junction plots (lattice nodes) with the finite jump (Ahlberg, Nilson and Walsh, 1972). The pieces of a spline converge in the nodes in an optimal way, so that the values of the function and all its derivatives to the left and to the right would coincide, i.e. $f^{(k)}\left(x_{i}+0\right)=f^{(k)}\left(x_{i}-0\right), k=0,1,2 \ldots q, i=1 \ldots n$. Then $f(x), f^{\prime}(x), f^{\prime \prime}(x), \ldots$ become continuous functions throughout the whole interval $\left[x_{1} \ldots x_{n}\right]$.

At modeling continuous economic dynamics, the model accurately goes through the discrete nodes of the process; thus, an approximating polynomial with further interpolation of the function values at any plots between the nodes is obtained.

The following sequence of actions is suggested in the economics as the method of analysis of correlations by differentiation of spline models (Ilyasov, 2009):

1) Economic processes are analyzed for presence of correlation dependence;

2) Mathematical models of the economic dynamics are built by approximation with cubic splines (Ilyasov, 2008);

3) By differentiation of the obtained spline models, we obtain the models of the growth rate of the studied dynamics;

4) By visualization of the derivative spline models (Ilyasov, 2013) of the studied dynamics, we make conclusions about the presence of correlation and about the correspondence of the results of the analysis to the calculated correlation ratios.

\section{Results}

As the empirical base of the research, we take the economic indexes of the oil and gas sphere. At that, in one case we are going to consider the situation with strong correlative dependence, which is close to functional. In the other case, we will take two rows of dynamics, for which the correlation ratio is close to zero.

For example, multiple researches evidence the presence of strong correlation between export prices for natural gas and oil. Having analyzed the data on the average export prices for crude oil and natural gas in 2000-2012, we found that the correlation ratio between the said pair of prices is equal to $r=0.982301$. The obtained value of the correlation ratio proves strong correlation between average export prices for crude oil and natural gas, which is close to functional.

The next step is to build mathematical models of dynamics of export prices for oil and gas. We are going to carry out the modeling by approximation with cubic splines. 
Table 1. Export of crude oil and natural gas by the Russian Federation during 2000-2012

\begin{tabular}{ccccc}
\hline Years & \multicolumn{2}{c}{ Crude oil } & \multicolumn{2}{c}{ Natural gas } \\
\hline & Quantity, MT & $\begin{array}{c}\text { Average export price, } \\
\text { USD/BL }\end{array}$ & $\begin{array}{c}\text { Quantity, } \\
\text { BCM }\end{array}$ & $\begin{array}{c}\text { Average export price, } \\
\text { USD/TCM }\end{array}$ \\
\hline 2000 & 144.4 & 23.94 & 193.9 & 85.84 \\
2001 & 164.5 & 20.78 & 180.9 & 98.25 \\
2002 & 189.5 & 21.02 & 185.5 & 85.69 \\
2003 & 228.0 & 23.81 & 189.4 & 105.51 \\
2004 & 260.3 & 31.02 & 200.4 & 109.05 \\
2005 & 252.5 & 45.21 & 209.2 & 151.36 \\
2006 & 248.4 & 56.32 & 202.8 & 216.00 \\
2007 & 258.6 & 64.28 & 191.9 & 233.66 \\
2008 & 243.1 & 90.68 & 195.4 & 353.69 \\
2009 & 247.5 & 55.61 & 168.4 & 249.27 \\
2010 & 250.7 & 74.11 & 177.8 & 268.48 \\
2011 & 244.5 & 101.74 & 189.7 & 338.88 \\
2012 & 240.0 & 103.14 & 178.7 & 348.33 \\
\hline
\end{tabular}

The main property of splines, which allows proposing this approach for analysis of correlations, is their continuous nature and differentiability. By differentiation of spline functions, we obtain a more sensitive tool for analysis - the derivatives of the spline functions. Involvement of derivatives in the process of modeling trends of an economic process is more preferable rather than table (lattice) representation of data and use of such concepts only as finite differences, gains, growth rates, etc. The necessity of such an approach is obvious, as economic processes are indeed continuous and inertial.

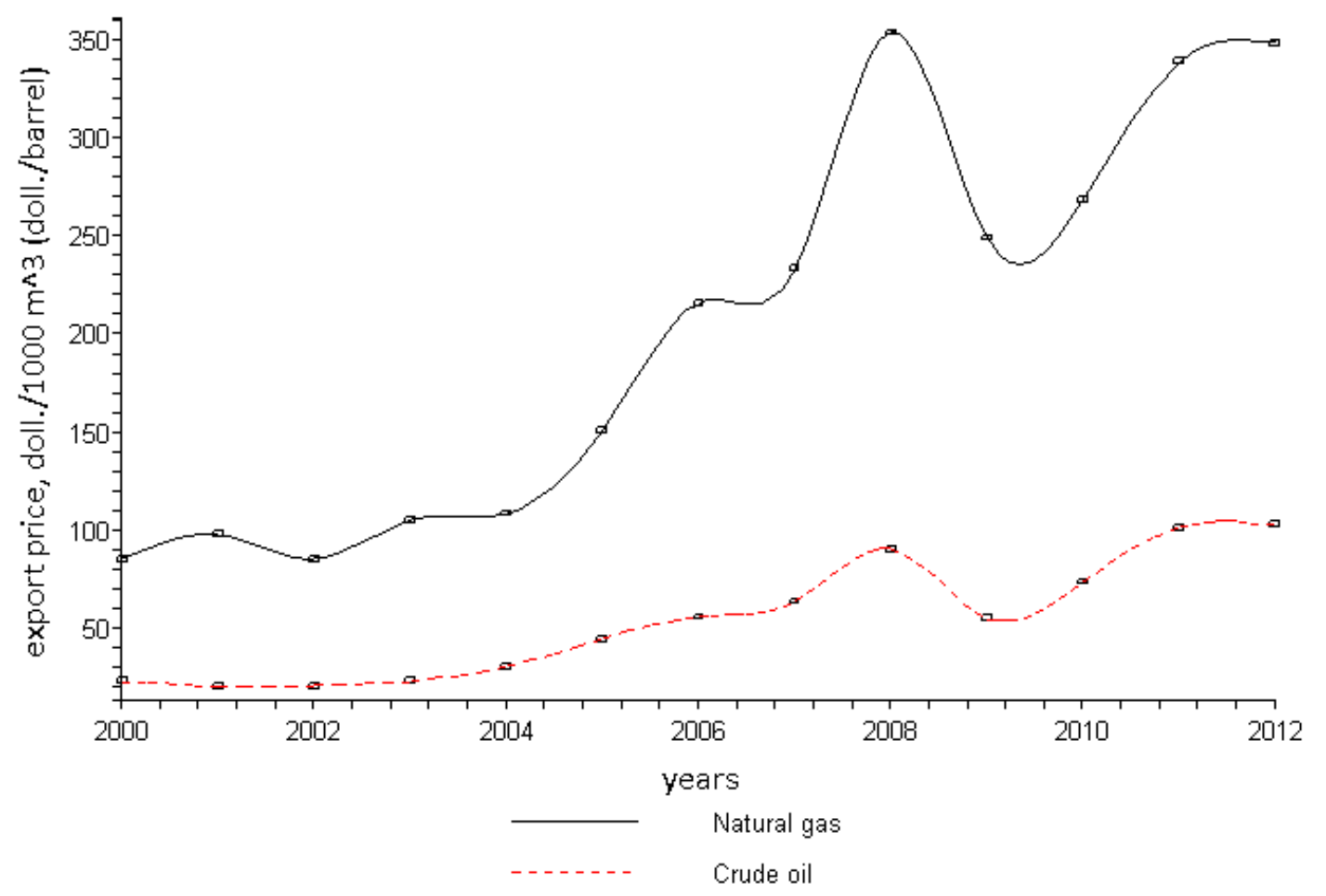

Figure 1. The dynamics of average export prices for natural gas and crude oil between 2000 and 2012. Approximation with cubic splines 
Comparison of the curves of dynamics of the export prices for natural gas and oil in Figure 1 represented by cubic splines shows that the prices for gas and the prices for oil demonstrate synchronous fluctuations, which evidences existence of stable correlation between the specified pair of prices. The previously found correlation ratio also confirms the high degree of association closeness.

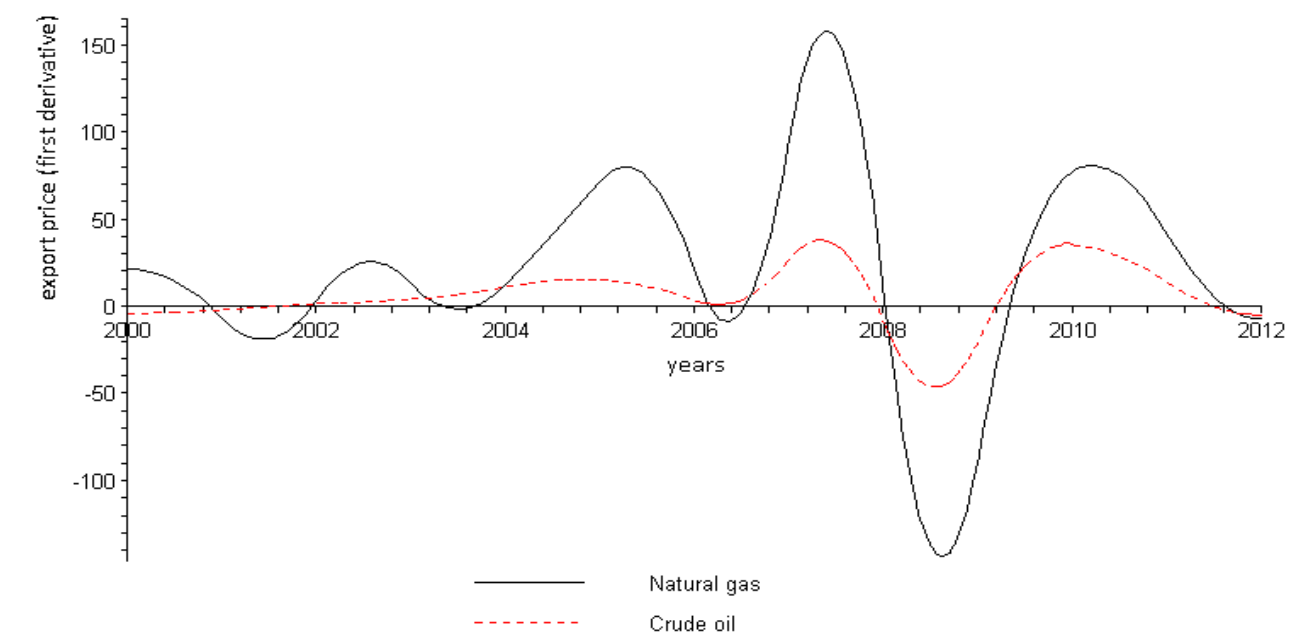

Figure 2. First derivatives of cubic splines approximating the trends of export prices for crude oil (the broken line) and natural gas (the full line). The data on prices are between 2000 and 2012

The next move is to compare the behavior of the first derivatives of the models representing the dynamics of export prices for natural gas and crude oil. The first derivative has rather logical economic content and meaning. It is a continuous trend of the process and indicates the rate of change of the studied index.

The dynamics of the price growth rate, as it is seen in Figure 2, also demonstrates synchronous and proportional amplitude of fluctuations. In this case, we do not see any considerable differences in the results of the correlation analysis based on both the correlation method and the method of comparison of the growth rate of the studied pair of prices - a strong positive correlation is observed.

More interesting situation is when results of the correlation analysis do not match the conclusions made as a result of comparison of the first two derivative spline models. As an example, let us consider the dynamics of the volumes of the natural gas and crude oil export from Russia during 2000-2012.

In this case, the correlation ratio equals to $r=0.156027$. With such a value of the correlation ratio, econometrics denies existence of any correlation between the studied dynamics rows.

The data in Table 1 demonstrate the complex nature of the studied dynamics - there are intervals here with both oppositely directed dynamics and synchronization of the growth directions. In this case, we cannot identify any regularities in the fluctuations of the studied dynamics using econometric methods.

We will build the models of the dynamics of volumes of the oil and gas export by approximation with cubic splines. Then, we will carry out transition to the models of their growth rate by differentiation of the spline models, which will allow detecting the "latent trends". Figure 3 demonstrates the asynchronous behavior of the fluctuations of the export volumes growth rate - as the volumes of oil export decrease, the volumes of gas export grow. In this case, two assumptions can be made:

1) Due to the interchangeability of oil and gas, decrease in the export of one of the energy sources is compensated with proportional increase in the volumes of export of the other one.

2) A chart of the first derivative spline model of the crude oil export volumes dynamics can be built by shifting the chart of the first derivative spline model of the natural gas export volumes dynamics one year backwards. 


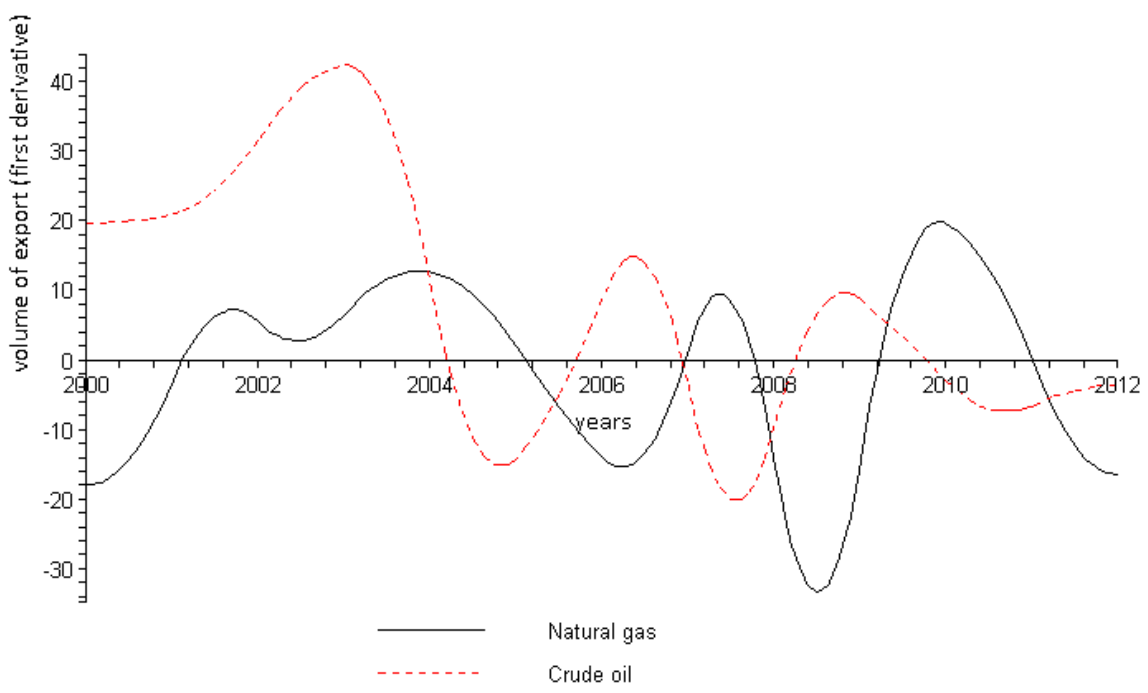

Figure 3. The first derivatives of cubic splines approximating trends of volumes of the crude oil and natural gas export from 2000 until 2012

In order to substantiate the first assumption, we will find the correlation ratio between the values of the first derivative spline models of the dynamics of volumes of the crude oil and natural gas export using the data of Table 2 . The obtained result $r=0.118777$ does not confirm the first assumption.

In order to verify the second assumption, we will find the correlation ratio between the values of the first derivative spline models of the dynamics of volumes of the crude oil and natural gas export shifted one year backwards. The obtained value $r=0.734616$ indicates quite close correlation between the volumes of the oil and natural gas export. Thus, the dynamics of the volumes of the natural gas export by the Russian Federation reacts to the decrease in volumes of crude oil export by proportional decrease with a delay of up to one year.

Probably, the revealed correlation could have been detected at calculation of the correlation ratio between the original values of dynamics of the volumes of the crude oil and natural gas export when shifting the latter one year backwards. However, in this case, we only obtain the value $r=0.286268882$. It is obvious that spline approximation and transition by differentiation to the models of the dynamics growth rate were efficient.

Table 2. Export of crude oil and natural gas by the Russian Federation during 2000-2012

\begin{tabular}{ccccc}
\hline Years & \multicolumn{2}{c}{ Crude oil } & \multicolumn{2}{c}{ Natural gas } \\
\hline & Quantity, MT & $\begin{array}{c}\text { Values of the first } \\
\text { derivatives of spline } \\
\text { models }\end{array}$ & $\begin{array}{c}\text { Quantity, } \\
\text { BCM }\end{array}$ & $\begin{array}{c}\text { Values of the first } \\
\text { derivatives of spline } \\
\text { models }\end{array}$ \\
2000 & 144.4 & 19.67 & 193.9 & -17.89 \\
2001 & 164.5 & 20.98 & 180.9 & -2.91 \\
2002 & 189.5 & 31.99 & 185.5 & 5.40 \\
2003 & 228.0 & 42.34 & 189.4 & 6.76 \\
2004 & 260.3 & 10.66 & 200.4 & 12.64 \\
2005 & 252.5 & -13.87 & 209.2 & 1.91 \\
2006 & 248.4 & 9.36 & 202.8 & -14.00 \\
2007 & 258.6 & -4.42 & 191.9 & 1.94 \\
2008 & 243.1 & -9.09 & 195.4 & -15.12 \\
2009 & 247.5 & 8.69 & 168.4 & -13.76 \\
2010 & 250.7 & -2.97 & 177.8 & 19.56 \\
2011 & 244.5 & -6.37 & 189.7 & -0.44 \\
2012 & 240.0 & -3.53 & 178.7 & -16.43 \\
\hline
\end{tabular}




\section{Discussion}

The modern approaches to the analysis of economic dynamics (Lemmens, Croux and Stremersch, 2012) require applying efficient tools, which would not be limited to detecting common regularities and correlations and would provide the researcher with the opportunity to detect also the "latent trends". This would allow to solve problems related to usage of the analysis results at planning and forecasting more efficiently, thus minimizing alternative losses of market players. One of such tools is the spline approximation approach (Zhanlav and Mijiddorj, 2010) to mathematical modeling of dynamics (Sarfraz, Zawwar and Nisar, 2010).

Among all the spline functions, the cubic splines were preferred, as they have such an attractive optimization property as internal optimality (Korn and Korn, 1973). This property of cubic splines is called the property of minimal curve or of minimal standard. For cubic spline functions $S_{\Delta}(y, x)$, it is expressed by the Holiday's theorem, which shows that spline buildup minimizes the integral:

$$
\int_{a}^{b}\left|f^{\prime \prime}(x)\right|^{2} d x \rightarrow \min
$$

Approximation with splines (Valenzuela and Pasadas, 2011; Kano, Fujioka and Martin, 2011) allowed obtaining qualitative models without the typical of econometrics procedures of smoothing and averaging the values of original dynamics (Aatola, Ollikainen and Toppinen, 2013; Benedictow, Fjaertoft and Lofsnes, 2013).

The special role of derivatives in mathematics and physics allows finding their efficient application also in economics at analysis, search for correlations, and forecast of economic behavior. The economic rationale of the first derivative is that it represents the trend or the rate of growth of an economic index; the second derivatives evidence changes in the growth rate, i.e. acceleration or slowdown of the index growth. Involvement of derivatives in the process of modeling the economic conjuncture allows seeing its trends much sooner and managing them, especially if we take into account the continuity and inertia of economic behavior. For qualitative description of processes, it is important to involve continuous analytical models, which have the properties of differentiability, smoothness, flatness, etc.

\section{Conclusion}

The method of correlations analysis suggested by the authors has a number of deficiencies, which can be eliminated in the long view:

1) There is no mechanism of correlation of values of the correlation ratios calculated for the derivative spline models with the correlation ratios for the original rows of dynamics;

2) The complexity of the method for an economist who does not have sufficient mathematical skills;

3) The greater labor input of the method if compared to the standard correlation analysis.

Despite the specified and other probable deficiencies, the authors believe that the value of the method resides in the sensitivity of this approach to local changes in the economic dynamics. It is also important for the modern economy to identify regularities in the fluctuations of the growth rate, and probably in the accelerations. The parameters of the interdependencies of economic indexes can vary greatly inside even a short interval; they cannot be qualitatively described with a single polynomial regression. As a response to it, spline representation of a parametric pattern of the correlation also demonstrates local reaction to the fluctuations of the factor attribute against the background of the general trend.

The continuity and differentiability of spline functions along with the open dynamic pattern also identify the "latent trends" of the process through the growth rate (the first derivative) and growth acceleration (the second derivative). The analysis of extremes of the first and second derivatives will be able to detect both event-related elements and small crises in the original dynamics.

\section{References}

Aatola, P., Ollikainen, M., \& Toppinen, A. (2013). Price determination in the EU ETS market: Theory and econometric analysis with market fundamentals. Energy Economics, 36, 380-395. http://dx.doi.org/10.1016/j.eneco.2012.09.009

Ahlberg, J., Nilson, J., \&. Walsh, L. (1972). The theory of splines and their applications. Academic Press, p. 318.

Benedictow, A., Fjaertoft, D., \& Lofsnes, O. (2013). Oil dependency of the Russian economy: An econometric 
analysis. Economic Modelling, 32, 400-428. http://dx.doi.org/10.1016/j.econmod.2013.02.016

Ilyasov R. (2013). Phase analysis of the cyclical share of natural gas in the structure of export of the Russian Federation. Polysubject Network Electronic Scientific Journal of Kuban State Agrarian University, 4, 88. Retrieved March 13, 2014, from http:// www.elibrary.ru/author_items.asp?authorid=537030

Ilyasov, R. (2008). Spline-analysis "thin" structures of interdependence of the export prices for natural gas and oil. Scientific-technical Bulletin of SPbSPU. Economic Science, 6(68), 348-352.

Ilyasov, R. (2009). Phase spline-analysis as a method of diagnostics of cyclicity in the economy. Modern High Technologies, 1, 32-36.

Kano, H., Fujioka, H., \& Martin, C. (2011). Optimal smoothing and interpolating splines with constraints. Applied Mathematics and Computation, 5, 1831-1844. http://dx.doi.org/10.1016/j.amc.2011.06.067

Korn, G., \& Korn, T. (1973). Mathematical Handbook for Scientists and engineers ditch. Moscow: Nauka. p. 832.

Lemmens, A., Croux, Ch., \& Stremersch, S. (2012). Dynamics in the international market segmentation of new product growth. International Journal of Research in Marketing, 1, 81-92. http://dx.doi.org/ 10.1016/j.ijresmar.2011.06.003.

Oh, H., Kim, J. G., \& Hong, W. (2008). The piecewise polynomial partition of unity functions for the generalized finite element methods. Computer Methods in Applied Mechanics and Engineering, 197(45-48), 3702-3711. http://dx.doi.org/10.1016/j.cma.2008.02.035

Pinto, H. (2011). The role of econometrics in economic science: An essay about the monopolization of economic methodology by econometric methods. The Journal of Socio-Economics, 40(4), 436-443. http://dx.doi.org/ 10.1016/j.socec.2011.04.011

Sarfraz, M., Zawwar, M., \& Nisar, A. (2010). Positive data modeling using spline function. Applied Mathematics and Computation, 7, 2036-2049.

Schoenberg, I. J. (1968). On the Ahlberg-Nilson extension of spline interpolation: The g-splines and their optimal properties. Journal of Mathematical Analysis and Applications, 21(1), 207-231.

Schoenberg, I. J. (1970). A second look at approximate quadrature formulae and spline interpolation. Advances in Mathematics, 4(3), 277-300.

Schoenberg, I. J. (1979). On Cardinal Spline Smoothing. North-Holland Mathematics Studies, 35(4), 383-407.

Schoenberg, I. J. (1983). A new approach to Euler splines. Journal of Approximation Theory, 39(4), 324-337.

Sekigawa, H. (2011). Computing the nearest polynomial with a zero in a given domain by using piecewise rational functions. Journal of Symbolic Computation, 46(12), 1318-1335. http://dx.doi.org/ 10.1016/j.jsc.2011.08.012.

Valenzuela, O., \& Pasadas, M. (2011). Fuzzy data approximation using smoothing cubic splines: Similarity and error analysis. Applied Mathematical Modelling, 5, 2122-2144. http://dx.doi.org/ 10.1016/j.apm.2010.11.046.

Wang, L., \& Yu, M. (2014). Favards theorem of piecewise continuous almost periodic functions and its application. Journal of Mathematical Analysis and Applications, 413(1), 35-46. http://dx.doi.org/ 10.1016/j.jmaa.2013.11.029

Zhanlav, T., \& Mijiddorj, R. (2010). The local integro cubic splines and their approximation properties. Applied Mathematics and Computation, 7, 2215-2219. http://dx.doi.org/ 10.1016/j.amc.2014.01.043

\section{Copyrights}

Copyright for this article is retained by the author(s), with first publication rights granted to the journal.

This is an open-access article distributed under the terms and conditions of the Creative Commons Attribution license (http://creativecommons.org/licenses/by/3.0/). 